\title{
Separation and reunion
}

\section{A short history of mind-body dualism}

\author{
Stephanie Mokrycke (Meds 2017), Hao Li (Meds 2016) \\ Faculty Reviewer: Dr Shelley McKellar, PhD (Department of History)
}

In a hospital, the body is divided into departments. One department for the ear, nose and throat, one for the eyes, one for the stomach and the intestines, one for the sexual organs, one for heart and blood vessels, and one for the soul, which is treated in the psychiatric wards.

\section{- Karl Ove Knausgaård, The Other Side of the Face}

As medical students trained with a mind for science, it is sometimes difficult to consider the human body as anything other than a series of anatomical parts. It is as if the human body is a great, complex machine that begins ticking early in the life of the embryo, and continues its autonomous functioning until the end, when system by system, the machine will slowly dissemble and finally lay itself to rest. The comparison of the human body to machine is called mechanization, and it is something that people have been doing since the $17^{\text {th }}$ century, the mechanical century. Thinking of this kind is closely linked to materialism-a philosophy that attributes all phenomena, including mental phenomena and consciousness, to be the result of material interactions-and largely informs the way we understand the brain and mind today. When materialism influenced the understanding of the brain and mind during the 17th century however, it posed a problem for Descartes, who was trying to accord the presence of a consciousness with the theological principles of the time. Cartesian mind-body dualism considered thought processes to be distinct from the body-machine, and it ascribed the thinking properties of the mind to an immortal and immaterial soul. The brain, specifically the pineal gland, was the seat of the soul. The soul was an individual's connection to the heavens, and therefore not reduced to the materiality of the body. ${ }^{1}$

While the consideration of the soul with respect to the brain and mind may seem strange to the scientific-minded medical student, it is not an alien concept in medicine. Consider the two specialties that deal primarily in the brain and mind, neurology and psychiatry. Materialism is recognizable in neurology: a neurologist locates aberrant anatomical lesions throughout the nervous system and is generally able to explain neurological disease on the basis of objective findings and sound physiological reasoning. Mental illnesses, on the other hand, are quite unique in that they are almost exclusively characterized by subjective symptomatology and rarely on discrete or objective findings. At the same time, we recognize today that mental disorders can manifest with bodily symptoms. Not unlike Cartesian dualism then, mental illnesses have required the framework of a conceptual separation of mind and body. Descartes' "soul" may not have disappeared from our mode of thinking after all, but instead, as Francis Azouvi suggests in an essay titled "Physique and Moral," the concept of the soul may have simply been replaced by the psychological. ${ }^{1}$

The division between brain and mind had not always been so apparent in medicine. It was not until the 19th century that medicine of the brain and mind truly diverged into the two separate specialties of neurology and psychiatry. As neuroanatomists were able to locate lesions related to specific diseases, the explanation for neurological illnesses became increasingly anatomical; likewise, the understanding of germ theory and use of microscopy enabled infectious disorders of the mind, such as neurosyphilis, to be understood and treated definitively. Yet, psychiatric illness that could not be placed in the anatomical or physiological realm remained, and the understanding of the etiology of those mental illnesses was still heavily influenced by the tremendous optimism for materialism at the time. ${ }^{2}$ It is not surprising then, that the various treatments offered at asylums were somatic in nature-aimed at correcting a flawed nervous system using methods such as hydrotherapy, electrical stimulation, and rest. ${ }^{3}$ But in the late 19th century to early 20th century, the understanding and treatment of mental disorders would begin to look to the soul, or as it would come to be known, the psychological.

Psychoanalysis has probably been most famed and subsequently defamed by the work of Sigmund Freud, although several other prominent neurologists, psychologists, and psychiatrists have made major contributions to the psychoanalytic movement and its resultant schools of thought. In the absence of disease-specific brain abnormalities, proponents of psychoanalysis recognized that some illnesses appeared to have no discernable organic basis, and theorized that psychological causes originating in childhood experiences and the unconscious were the root of mental disease. Treatment was thus focused on techniques such as dream interpretation, free association, or transference, which did not aim to alter a physical aberration related to mental disease, but rather to subjectively explore the mind. The immaterial nature of a patient's "psychology", therefore, was investigated and manipulated in order to diagnose, treat, and sometimes cure mental disorders.

At the turn of the 20th century, the connection between the body and mind in the history of medicine was not well defined. Both brain and mind had been implicated as the cause for mental and somatic disorders, and manipulating both the body and the mind had been considered as treatment regimens. Today, it is clear that a more modern understanding of the brain and mind incorporates a 
unity between the two; thus over the course of the 20th century, we would come to see the divergent relationship of the brain and mind converging once again in medicine.

The union of the structural and abstract theories of the brain and mind began in the late 19th century, but did not come to popular attention until the 1930s. ${ }^{4}$ In 1935, the first frontal lobotomy was performed in Lisbon, Portugal by Egas Moniz, a professor of neurology, and Almeida Lima, a surgeon. The principle of the surgery was based on research that showed the association of the frontal lobes of the brain with temperament and behavioural control. Proponents of lobotomy surgery believed that dysfunctional behaviour was caused by pathological material located in the frontal lobes. Ablating the pathological tracts of the frontal lobe was the goal of the therapy. The side effects from the procedures performed were numerous: while some patients became more docile after the operation, others experienced an increase in impulsive behaviour, lack of initiative, and reduced ability to think or plan clearly. Effectiveness of the procedure was strongly debated, and it was popularized by the media as one of the greatest medical blunders of the time; however, though infamous, frontal lobotomy has contributed greatly to the study of mind and behaviour, particularly by demonstrating the role of neuroanatomy in human psychology.

Three years after the first lobotomy surgery was performed in Portugal, two Italian physicians, Lucio Bini and Ugo Cerletti, completed the first session of electroconvulsive therapy (ECT) on a catatonic patient. This initial use of ECT relied on the understanding that electrical stimuli could be used to induce seizures in order to treat psychiatric abnormalities. The 1938 treatment was a success, and even though the exact mechanism remains elusive, ECT is used today for treatment of major depressive disorders in select patients. ${ }^{6}$ The use of ECT was closely followed by treatments established in psychopharmacology. The introduction of psychopharmacology began in the early 1950s, with the first wave of psychotropic medications providing a treatment option for previously untreatable psychiatric conditions. ${ }^{7}$ The therapies were based on an understanding that neurochemical imbalances in the brain can precipitate psychological disorders, and offered yet another physiological mode of viewing the interaction between brain function and mental phenomena.

Today, it is recognized that a combination of psychotherapy, medical therapy, and even surgical therapy can be effective in treating mental illnesses. ${ }^{8}$ But unlike a pathogen being eradicated by an antibiotic, or a structural defect being corrected, individual patient responses to psychiatric treatments are unique; that is, those that respond to some therapies may not respond to others, and the response cannot always reliably be predicted. In light of these enduring limitations of modern medicine, it causes one to question how far we have come from the lobotomy surgeons of the past.

The scientific investigation of the brain and mind has made great strides over the past century in order to advance the study of mental disorders. Improvements in surgical techniques and imaging modalities will only act to further advance the understanding of the brain and mind. The trouble of course is that there is still much to understand before we can arrive at a complete understanding of mental disorders. The Diagnostic and Statistical Manual of Mental Disorders (DSM) is just one example of how psychiatric medicine is still largely reliant on subjective symptomatology. Despite the impressive research in functional neuroanatomy and neurobiology being undertaken, there still does not exist a laboratory test, or diagnostic imaging that can reliably identify psychiatric illness. This may be because the brain and mind is one of the most complicated areas of medicine that humans have attempted to understand. No other anatomical structure contains such a vast amount of information transmission to decode. If one considers that the knowledge we currently have may represent only the tip of the iceberg, the fact that many of the treatment regimens we use today work for patients can seem miraculous. So if the discovery of how the mind works is still ahead of us, we must somehow create a model of the brain and mind that does not rely on the purely physical or the purely psychological. It seems that despite our vast increase in knowledge since the time of Descartes, not much has changed in the fundamental theory of body and soul.

In A Scandalously Short Introduction to the History of Medicine, Jaclyn Duffin warns us that the over-mechanization of the $19^{\text {th }}$ century risked the loss of insight into the mind-body connection. ${ }^{2}$ But increasingly the culture of medicine has been reaching back to incorporate the subjective patient experience to include a spiritual, cultural, and psychological understanding of illness. In the $21^{\text {st }}$ century, perhaps a rigorous pursuit of knowledge will not cause us to lose sight of the mind-body connection, but instead, enlighten us with a greater appreciation for the body and the soul. This happy marriage of objective science and subjective patient experiences may be the aspect of modern medicine that allows for a deeper understanding of the mind-body connection, and ultimately, creates a culture of medicine that reserves a space for the soul.

\section{REFERENCES}

1. Potter P, Wright JP (eds.). Psyche and soma: physicians and metaphysicians on the mind-body problem from antiquity to enlightenment. New York: Oxford University Press; 2000. Print.

2. Duffin J. History of Medicine: A scandalously short introduction. Toronto: University of Toronto Press; 2010. Print.

3. Holtzman E. A home away from home. Monitor on Psychology. 2012 Mar;43(3):24.

4. Annenberg Learner. History of Psychology: Contemporary foundations [Internet]. St. Louis (MO): Annenberg Learner; 2001 [cited 2014 Nov 29]. http://www.learner.org/series/discoveringpsychology/history/ history_nonflash.html

5. Kucharski A. History of frontal lobotomy in the United States, 19351955. Neurosurgery. 1984 Jun;14(6):765-72.

6. Kalapatapu RK, Chang AK, Windle ML, Popeo DM. Electroconvulsive therapy [Internet]. Medscape; 2012 Jun 26 [cited 2014 Nov 29]. Available from: http://emedicine.medscape.com/article/1525957-overview.

7. Edward K, Alderman C. Psychopharmacology: practice and contexts. South Melbourne Victoria: Oxford University Press; 2013. 3-24 p.

8. Somers J, Querée M. Cognitive behavioural therapy. Vancouver: Simon Fraser University; 2007. 15-23, 45-51 p. 